\title{
ON THE POSTULATES AND LAWS OF ELECTROMAGNETIC THEORY IN STATIONARY CONTINUOUS MEDIA
}

Burak POLAT (burakpolat@beykent.edu.tr)

Beykent University, Faculty of Engineering \& Architecture, Department of Electronics and Communications Engineering, Istanbul, Turkey

\begin{abstract}
The postulates and laws that constitute the foundation of classical electromagnetic theory in stationary continuous media are reviewed and discussed from the author's perspective for a clear and precise comprehension in electrical engineering education.
\end{abstract}

Keywords: $\quad$ Electromagnetic fields, Maxwell equations, electromagnetics education, stationary media. 


\section{HAREKETSIZ SÜREKLİ ORTAMLARDA ELEKTROMANYETIZMA KURAMININ POSTÜLA VE YASALARI ÜZERINE}

Burak POLAT (burakpolat@beykent.edu.tr)

Beykent Üniversitesi, Mühendislik - Mimarllk Fakültesi, Elektronik ve Haberleşme Mühendisliği Bölümü, İstanbul, Türkiye

\section{ÖZET}

$\mathrm{Bu}$ çalı̧̧mada hareketsiz sürekli ortamlar için klasik elektromanyetizma kuramının esasını oluşturan postülalar, yasalar ve ilkeler literatürden atıflarla özgün bir yaklaşımla irdelenmiştir. Ortaya konan bakış açısının, elektrik-elektronik mühendisliği eğitiminin temelini oluşturan elektromanyetizma kuramının olabildiğince net ve doğru şekilde anlaşılmasına katkıda bulunabilmesi hedeflemektedir.

Anahtar Kelimeler: Elektromanyetik alanlar, Maxwell denklemleri, elektromanyetizma eğitimi, hareketsiz ortamlar 


\section{INTRODUCTION}

Classification and establishment of a methodology constitute the essence of scientific thinking. With this in mind we suggest the flowchart in Figure 1 to draw a general strategy for consecutive steps in analytical investigation of electromagnetic phenomena. The first block describes the construction of the physical model. One starts with the postulates and field equations (laws) of stationary (or moving) media which are combined with constitutive relations that describe the electromagnetic behavior of material media. Next, complementary postulates and laws which serve as the bridge that meets electromagnetism with other branches of physics (such as mechanics, thermodynamics, elasticity, acoustics, physiology, etc.) are involved so that the physical model is uniquely constructed. Many laws, principles, theorems or relations follow as an outcome of these fundamental equations immediately. Finally, the projection of the postulates on field behavior in presence of any physical singularity, such as boundary/transition conditions, initial conditions, the radiation condition, the edge condtions or other conditions specific to the problem, such as symmetry or periodicity, should be involved. The next block is the construction of the mathematical model. By incorporating all field relations, after successive decoupling and elimination operations, one obtains a mixed boundary value problem for the field quantities under investigation. This should be followed with the proof of the wellposedness of the associated boundary value problem under a most general parametrization. The following block is the derivation of a solution. To simplify the problem adequately, no physical constraint or approximation on the physical and geometrical parameters should be missed. Next, the methodology that complies with our purposes should be adopted. At this stage one may apply exactly analytical, asymptotic, purely computational or hybrid methods. The final block is the verification of the obtained analytical or numerical solution. 
First, the consistency of the solution should be checked in itself over various general mathematical/physical properties and principles such as periodicity, casuality, stability, reciprocity, duality, energy conservation, and so on. This should be followed by a numerical comparison between different solutions derived by alternative methods to estimate accuracy or convergence property over various parameters as a benchmark test. Satisfactory results herald a consistent analytical/numerical solution for the boundary value problem. Once the mathematical result is also verified by measurement data, our mathematical model can be called consistent with physical behavior as well. In case of any failure at the verification process each consequent step on the flowchart should be checked over.

In this paper the axiomatic structure of classical electromagnetic theory in stationary continuous media is studied from the author's perspective for educational purposes. The focus is on the postulates and laws of stationary continuous media which fall into the first block on the flowchart. 


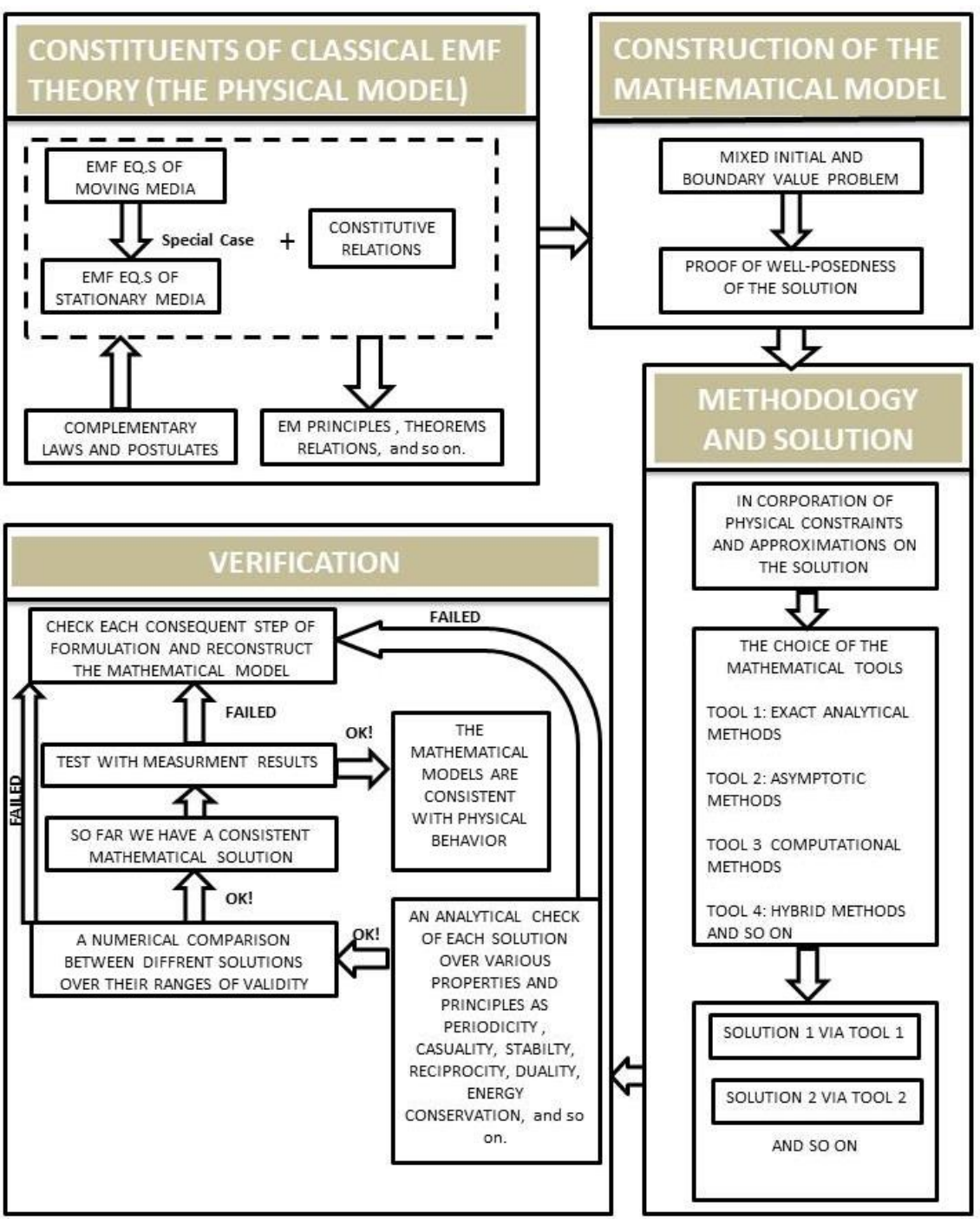

Figure 1. A Flowchart of Analytical Investigation of Electromagnetic Phenomena

\section{POSTULATES ON ELECTRICAL CHARGE}

Rougly speaking, the discipline of classical electromagnetism studies basically the relation between electrical and magnetic sources and the fields they generate as well as their connection with quantities from other disciplines of physics. To understand and characterize the concepts of this field theory, one must get down to 
the level of electric charge. The experimental evidences on the study of electrical charge pioneered by Cavendish, Robison, Coulomb, Millikan till date reveal that its behaviour can be summoned up under the following three postulates:

Postulate 1: There exists two kinds of electrical charges: a positive charge and a negative charge. The "plus" and "minus" signs rests on the fact that their effects tend to cancel one another.

Postulate 2: Electrical charge is conserved, it cannot be created or destroyed; in the sense that whenever any positive charge appears, an equal amount of negative charge also appears. Conversely, whenever any positive charge disappears, an equal amount of negative charge also disappears. Thus the algebraic sum of all charges is constant in any isolated system. This postulate is actually assumed to hold for the entire universe, which is called "global conservation of charge".

Postulate 3: All charges are integral multiples of the electronic charge, whose magnitude is given $e=1.60 \times 10^{-19}$ Coulomb. The study of subatomic particles (quarks) with charges which are fractions of $e$ are beyond the interest of classical electromagnetic theory.

As a good reference of the nature and behaviour of electrical charge as well as its experimental tests till date one may refer to the papers in the special issue in [1] (see also [2]). These three postulates on electrical charge are available in many references including [3, p.45], [4, p.12], [5, p.11]. It should be pointed out that, unlike the traditional approach, we consider Coulomb (or Lorentz) Law as a "complementary postulate" and do not include as a fourth postulate on electric charge at this stage. 


\section{TRANSITION FROM PARTICLE CHARGES TO MACROSCOBIC CHARGE DENSITY}

The second step in deriving a macroscobic field theory is to establish the transition from discrete electrical charges to a continuous distribution. Let us quote from [5, Sect.2.2] and [6, Sect.1.2.1] where the reasoning and validity of this transition is clearly described:

"Obviously, the requirement for a macroscobic field theory is associated with the fact that it is impossible to calculate the effect of each (free or bound) electric charge in a given system. Therefore a macroscobic volume charge density function defined at a point $\mathrm{P}$ as $\rho=\lim _{\Delta \vartheta \rightarrow \varepsilon} \Delta q / \Delta \vartheta$, where $\varepsilon$ is a small volume that shrinks onto the point $\mathrm{P}$ in the limit and $\Delta q$ is the total amount of charge in $\Delta \vartheta$. Since a point theoretically having zero volume cannot contain a physically finite sized electron, and the physical fact that the actual charge density must vary because of the thermal agitation of electrons and protons, it can be inferred that such a limit definition is inconsistent with the discreteness of electronic charge. The linear dimensions of the electron and proton, when described in classical terms, are estimated to be of the order of $10^{-15}[\mathrm{~m}]$, these regions occupied by these particles are tiny and comparatively isolated; moreover, their locations are continually changing. However, from a practical point of view, this does not pose a real difficulty. One can easily choose the volume $\varepsilon$ to be quite negligable in comparison to the relatively large volumes of interest in a macroscobic theory and still be assured of its being sufficiently large on the atomic (or microscobic) scale of electrons. The volume $\varepsilon$ may, in fact, contain an immense number of discrete charges. In a metal, for example, the free electron density is typically of the order of $10^{29}$ [electrons $/ \mathrm{m}^{3}$ ] . An elemental cube with sides as small as a thousand angstroms (i.e., $\left.10^{3}[\mathrm{~A}]=10^{-7}[\mathrm{~m}]\right)$ and thus a volume $\varepsilon=10^{-21}\left[\mathrm{~m}^{3}\right]$, contains 
$10^{8}=100$ millon electrons! Yet the size of the elemental cube ( $\left.10^{3}[\stackrel{\circ}{\mathrm{A}}]\right)$ is still quite negligable in comparison to the sizes of most electronic devices. Thus, any inaccuracies resulting from the assumption of a smooth charge distribution with a finite density are negligable. The advantage of a description of electromagnetic theory in terms of charge density is thus clear. The field calculated from a macroscobic charge density is, of course, correspondingly an "average" field; it is what would be found by a laboratory scale measurement."

One may also refer to [7, Sect.6.6] for the derivation of macroscobic Maxwell's equations by averaging each field quantity in microscobic Maxwell's equations in the distributional sense.

\section{ELECTROMAGNETIC FIELD EQUATIONS OF STATIONARY CONTINUOUS MEDIA}

Now that the link between microscobic and macroscobic field quantities is discussed, one may introduce the well-known Maxwell's Equations [8] as a postulate. Regarding cases when Maxwell's equations fail to explain electromagnetic phenomena the reader may refer to the survey paper [9].

Postulate 4: Macroscopic electromagnetic phenomena of stationary continuous material media are governed by the Maxwell's equations

$$
\begin{gathered}
\operatorname{curl} \vec{E}(\vec{r} ; t)+\frac{\partial}{\partial t} \vec{B}(\vec{r} ; t)=\overrightarrow{0}, \operatorname{curl} \vec{H}(\vec{r} ; t)-\frac{\partial}{\partial t} \vec{D}(\vec{r} ; t)=\vec{J}_{C}(\vec{r} ; t) \quad(1 \mathrm{a}, \mathrm{b}) \\
\operatorname{div} \vec{D}(\vec{r} ; t)=\rho_{f}(\vec{r} ; t), \operatorname{div} \vec{B}(\vec{r} ; t)=0 \quad(1 \mathrm{c}, \mathrm{d})
\end{gathered}
$$

or equivalently the integral set

$$
\oint_{\partial S} \vec{E} \cdot d \vec{c}+\frac{d}{d t} \int_{S} \vec{B} \cdot d \vec{S}=\overrightarrow{0}, \oint_{\partial S} \vec{H} \cdot d \vec{c}+\frac{d}{d t} \int_{S} \vec{D} \cdot d \vec{S}=\int_{S} \vec{J}_{C} \cdot d \vec{S}
$$


$\oint_{\partial \vartheta} \vec{D} \cdot d \vec{S}=\int_{\vartheta} \rho_{f} d \vartheta, \oint_{\partial \vartheta} \vec{B} \cdot d \vec{S}=0$

where the field quantities are defined in MKSA units as follows:

$\vec{E}(\vec{r} ; t):$ Electrical field vector $[\mathrm{V} / \mathrm{m}]$

$\vec{B}(\vec{r} ; t)$ : Magnetic induction density field (or magnetic displacement field) $\left[\mathrm{Wb} / \mathrm{m}^{2}\right]$ (or $[\mathrm{T}]$ )

$\vec{H}(\vec{r} ; t)$ : Magnetic field $[\mathrm{A} / \mathrm{m}]$

$\vec{D}(\vec{r} ; t)$ : Displacement density field (or electrical displacement field) $\left[\mathrm{C} / \mathrm{m}^{2}\right]$

$\vec{J}_{C}(\vec{r} ; t)$ : Conduction current density $\left[\mathrm{A} / \mathrm{m}^{2}\right]$

$\rho_{f}(\vec{r} ; t):$ Free charge density $\left[\mathrm{C} / \mathrm{m}^{3}\right]$

For a complete description of material media we also involve the closed form constitutive relations

$\vec{D}=\vec{f}_{d}(\vec{E} ; \vec{H})=\varepsilon_{0} \vec{E}+\vec{P}^{e}, \vec{B}=\vec{f}_{b}(\vec{E} ; \vec{H})=\mu_{0} \vec{H}+\vec{P}^{m}, \vec{J}_{C}=\vec{f}_{C}(\vec{E} ; \vec{H})$

with the additional field quantities

$\varepsilon_{0} \cong(1 / 36 \pi) \times 10^{-9}:$ Dielectric permittivity of free space $[\mathrm{F} / \mathrm{m}]$

$\mu_{0} \cong 4 \pi \times 10^{-7}$ : Magnetic permittivity of free space $[\mathrm{H} / \mathrm{m}]$

$\vec{P}^{e}(\vec{r} ; t)$ : Electrical polarization density field $\left[\mathrm{C} / \mathrm{m}^{2}\right]$

$\vec{P}^{m}(\vec{r} ; t)$ : Magnetic polarization density field $\left[\mathrm{Wb} / \mathrm{m}^{2}\right]$

and $\vec{f}_{d}, \quad \vec{f}_{b}, \vec{f}_{C}$ being suitable arbitrary functions that may characterize material media.

The presence of electrical and magnetic polarization vectors $\vec{P}^{e}$ and $\vec{P}^{m}$ are assumed to characterize (or include) all types of polarization mechanisms observable in any material medium. The Maxwell's 
equations in point form in (1a-d) can be stated in words briefly as follows:

"The curl of the electrical field at a point equals to the time rate of decrease of the magnetic displacement vector at that point." (also called Faraday's Law)

"The curl of the magnetic field at a point equals to the total electrical current density at that point." (also called Ampere's Law)

"The divergence of the electrical displacement vector at a point is equal to the electrical volume charge density at that point" (also called Law of Conservation of Electrical Flux)

"The divergence of the magnetic displacement vector at any point is equal to zero" (also called Law of Conservation of Magnetic Flux).

The present time notation of Maxwell equations is introduced by Heaviside, who also developed the modern vector operators. (see. [10]). Next we shall make a number of general remarks on this system of equations:

Remark 1: It should be mentioned that not all quantities of an electromagnetic space are supposed to vary with both space and time simulatenously provided that at least one of the quantities in (1a-d) is assumed a function of time.

Remark 2: By the term "stationary media" it should be understood that there are no convention currents, which means that there is no material displacement! This description conforms to the Hertzian formulation of electrodynamics for moving bodies (cf. [11] and the references cited therein), while main stream scientists who treat moving electromagnetic media in the context of special/general relativity invoke convection currents into the Maxwell's equations by summing up with conduction current (density) term. However, in presence of a point charge in arbitrary motion, the partial time 
derivatives $\frac{\partial}{\partial t}$ in the Maxwell equations fail to characterize the actual physical picture by themselves. (see [12], [13]).

Remark 3: There are two groups of actors in any field theory. These are "sources" and "fields". However, their descriptions are not unique. It depends to the representation one chooses. Some fields may involve source terms (which is the case with constitutive relations) and some sources can be constructed via field terms (called "equivalent sources"). Such an interpretation yields that one cannot talk about a unique presentation of the Maxwell's equations. In that regard the set (1a-d) is also called the "indefinite" form and alternative representations are given in the following section.

Remark 4: Obtaining field quantities from given sources and medium properties is called an "analysis" problem, while the opposite case is called a "synthesis" or a "design" problem. Either it is an analysis or a design problem, the actual solution can be obtained only when the problem is "well-posed" in the sense of Hadamard, i.e., a solution exists, the solution is unique, the solution depends continuously on data.

Remark 5: It is seen that dynamic electrical phenomena are modelled fundamentally via divergence and curl operations in space and first order differentiation operator in time. Of course, many more operators will be involved when we exercise on these equations, but still the mentioned operators are indispensible and their physical interpretations are of fundamental importance.

Remark 6: It is not always the case for an arbitrary vector $\vec{A}(\vec{r} ; t)$ that $\operatorname{curl} \vec{A}(\vec{r} ; t) \perp \vec{A}(\vec{r} ; t)$ or $\vec{A}(\vec{r} ; t) \| \frac{\partial \vec{A}}{\partial t}(\vec{r} ; t)$. Therefore one should not draw conclusions from (1a) or (1b) such as $\vec{E}(\vec{r} ; t) \perp \vec{B}(\vec{r} ; t)$ or 
$\vec{H}(\vec{r} ; t) \perp \vec{D}(\vec{r} ; t)$ or $\vec{J}_{C}(\vec{r} ; t)$ in any case regardless of the properties of a medium. (cf. [14], [15] and the references cited therein).

Remark 7: One common property of divergence, curl and partial time derivative operators is their linearity. On the other hand, all source quantities in Maxwell's equations are of the first order. These properties directly addresses that the Postulate 4 inherently involves "the principle of superposition".

This property can be stated as follows: Consider that we have two sets of sources $\left(\rho_{f}^{I}, \vec{J}_{C}^{I}\right)$ and $\left(\rho_{f}^{I I}, \vec{J}_{C}^{I I}\right)$. Then the total field at any point when the two sources exist simultaneously is the vector sum of the (partial) fields generated when they appear by themselves in the same medium:

$$
\vec{E}\left[\left(\rho_{f}^{I}, \vec{J}_{C}^{I}\right) \cup\left(\rho_{f}^{I I}, \vec{J}_{C}^{I I}\right)\right]=\vec{E}\left[\left(\rho_{f}^{I}, \vec{J}_{C}^{I}\right)\right]+\vec{E}\left[\left(\rho_{f}^{I I}, \vec{J}_{C}^{I I}\right)\right]
$$

and similarly for the other three field components. However, it should not be inferred that the actual coupling mechanism between the two sets of sources is disregarded when one treats them individually.

Remark 8: The most critical components in the dynamic field relations are the electrical and magnetic displacement current densities $\frac{\partial \vec{D}}{\partial t}(\vec{r} ; t)$ and $\frac{\partial \vec{B}}{\partial t}(\vec{r} ; t) . \frac{\partial \vec{D}}{\partial t}$ has the same unit as the electrical conduction current density function $\vec{J}_{C}$, which implies that this term also signifies a certain mechanism of current flow. A current that is generated by a dynamic field as opposed to by motion of electrons! This groundbreaking contribution of Maxwell to electromagnetism denotes that the flow of current (or of the associated energy) may be maintained by the displacement field at any point where conduction or convection currents are cut off 
abruptly in a dynamic system, and is closely associated with the radiation mechanism of antennas.

\section{ON ALTERNATIVE REPRESENTATIONS OF THE FIELD EQUATIONS}

Which type of analogy is there between electrical and magnetic field quantities? Two different viewpoints discussed in [16, Sect.104] yield opposite results:

1. By looking at the mathematical (dual) structure of the field equations (1a-d) it can be inferred that $\vec{E}$ and $\vec{D}$ can be correlated to $\vec{H}$ and $\vec{B}$, respectively.

2. On the other hand, the definitions of $\vec{E}$ and $\vec{B}$ (based on a test charge in Coulomb and Lorentz Force Laws) make no reference to electric and magnetic properties atoms or molecules and holds equally well both macro- and microscobically. By contrast, the microscobic definitions of $\vec{D}$ and $\vec{H}$ in (3a,b) involve $\vec{P}^{e}$ and $\vec{P}^{m}$, and hence depend on atomic or molecular properties; the step from the micro- to macroscobic definitions involves taking averages over multitudes of atoms. From this standpoint $\vec{E}$ and $\vec{D}$ are correlated respectively to $\vec{B}$ and $\vec{H}$.

Another argument is which pair of the four electric and magnetic field quantities is more meaningful, more descriptive and more convenient in specific computations. For this purpose let us introduce the following polarization source quantities:

$\rho_{P}^{e}(\vec{r} ; t)=-\operatorname{div} \vec{P}^{e}(\vec{r} ; t)$ : Electrical polarization (or bounded) charge density $\left[\mathrm{C} / \mathrm{m}^{3}\right]$

$\rho_{P}^{m}(\vec{r} ; t)=-\operatorname{div} \vec{P}^{m}(\vec{r} ; t)$ : Magnetic polarization (or bounded) charge density $\left[\mathrm{Wb} / \mathrm{m}^{3}\right]$ 
$\vec{J}_{P}^{e}(\vec{r} ; t)=\frac{\partial}{\partial t} \vec{P}^{e}(\vec{r} ; t)$ : Electrical polarization (or bounded) current density $\left[\mathrm{A} / \mathrm{m}^{2}\right]$

$\vec{J}_{P}^{m}(\vec{r} ; t)=\frac{\partial}{\partial t} \vec{P}^{m}(\vec{r} ; t)$ : Magnetic polarization (or bounded) current density $\left[\mathrm{V} / \mathrm{m}^{2}\right]$

$\vec{J}_{V O}^{e}(\vec{r} ; t)=\left(1 / \varepsilon_{0}\right) \operatorname{curl} \vec{P}^{e}(\vec{r} ; t) \quad$ Electrical vortex current density [V/ $\left.\mathrm{m}^{2}\right]$

$\vec{M}(\vec{r} ; t)=\left(1 / \mu_{0}\right) \vec{P}^{m}(\vec{r} ; t):$ Magnetization vector $[\mathrm{A} / \mathrm{m}]$ with $\vec{B}=\mu_{0}(\vec{H}+\vec{M})$

$\vec{J}_{V O}^{m}(\vec{r} ; t)=\left(1 / \mu_{0}\right) \operatorname{curl} \vec{P}^{m}(\vec{r} ; t)=\operatorname{curl} \vec{M}(\vec{r} ; t) \quad\left[\mathrm{A} / \mathrm{m}^{2}\right]$ Magnetization (or magnetic vortex) current density

while $c_{0}^{2}=1 /\left(\varepsilon_{0} \mu_{0}\right)$. The notations $\vec{J}_{P}^{e, m}, \vec{J}_{V O}^{e, m}$ belong to the present author. Next, we outline the four possible pairings as follows:

\section{Form A1.}

Fundamental Fields: $(\vec{E}, \vec{B})$

Fundamental Sources: $\left(\rho_{f}, \vec{J}_{C}\right),\left(\rho_{P}^{e}, \vec{J}_{P}^{e}\right), \vec{J}_{V O}^{m}$

$\operatorname{curl} \vec{E}+\frac{\partial \vec{B}}{\partial t}=\overrightarrow{0}, \operatorname{curl} \vec{B}-\frac{1}{c_{0}^{2}} \frac{\partial \vec{E}}{\partial t}=\mu_{0}\left(\vec{J}_{C}+\vec{J}_{P}^{e}+\vec{J}_{V O}^{m}\right)$

$\operatorname{div} \vec{E}=\left(1 / \varepsilon_{0}\right)\left(\rho_{f}+\rho_{P}^{e}\right) \quad, \operatorname{div} \vec{B}=0$

This representation is called Amperian model in literature as emphasized in [16, p.367] and is especially suitable in particle dynamics where the Lorentz Force comes into play and in relativistic electrodynamics for applying Lorentz transformations. 


\section{Form A2.}

Fundamental Fields: $(\vec{E}, \vec{H})$

Fundamental Sources: $\left(\rho_{f}, \vec{J}_{C}\right),\left(\rho_{P}^{e}, \vec{J}_{P}^{e}\right),\left(\rho_{P}^{m}, \vec{J}_{P}^{m}\right)$

$\operatorname{curl} \vec{E}+\mu_{0} \frac{\partial \vec{H}}{\partial t}=-\vec{J}_{P}^{m}, \operatorname{curl} \vec{H}-\varepsilon_{0} \frac{\partial \vec{E}}{\partial t}=\vec{J}_{C}+\vec{J}_{P}^{e}$

$\operatorname{div} \vec{E}=\left(1 / \varepsilon_{0}\right)\left(\rho_{f}+\rho_{P}^{e}\right) \quad, \operatorname{div} \vec{H}=\left(1 / \mu_{0}\right) \rho_{P}^{m}$

This model is particularly convenient in electromagnetic wave propagation theory in MKSA units since it is suitable to describe the intrinsic impedance of a medium and power flow in terms of $\vec{E}$ and $\vec{H}$ fields. (see also [16, p.367], [17, p.xiii]).

\section{Form A3.}

Fundamental Fields: $(\vec{D}, \vec{B})$

Fundamental Sources: $\left(\rho_{f} ; \vec{J}_{C}\right), \vec{J}_{V O}^{e}, \vec{J}_{V O}^{m}$

$\operatorname{curl} \vec{D}+\varepsilon_{0} \frac{\partial \vec{B}}{\partial t}=\varepsilon_{0} \vec{J}_{V O}^{e}, \operatorname{curl} \vec{B}-\mu_{0} \frac{\partial \vec{D}}{\partial t}=\mu_{0}\left(\vec{J}_{C}+\vec{J}_{V O}^{m}\right)$

$\operatorname{div} \vec{D}=\rho_{f}, \operatorname{div} \vec{B}=0$

\section{Form A4.}

Fundamental Fields: $(\vec{D}, \vec{H})$

Fundamental Sources: $\left(\rho_{f}, \vec{J}_{C}\right),\left(\rho_{P}^{m}, \vec{J}_{P}^{m}, \vec{J}_{V O}^{e}\right)$

$\operatorname{curl} \vec{D}+\frac{1}{c_{0}^{2}} \frac{\partial \vec{H}}{\partial t}=\varepsilon_{0}\left(\vec{J}_{V O}^{e}-\vec{J}_{P}^{m}\right), \operatorname{curl} \vec{H}-\frac{\partial \vec{D}}{\partial t}=\vec{J}_{C}$

$\operatorname{div} \vec{D}=\rho_{f}, \operatorname{div} \vec{H}=\left(1 / \mu_{0}\right) \rho_{P}^{m}$ 
Forms A3 and A4 have not attracted much interest in the history of electromagnetism. Finally let us quote the views of C.T. Tai [18] on the argument:

"As long as Maxwell's equations are written in their indefinite form (1a-d) all these variants are equally acceptable. The reason for this strong statement is that once the constitutive relations are known or specified all of them reduce to one definite form, and it is the final form of Maxwell's equations with the constitutive relations that provide us a working model. In other words, it is meaningless to speak of the solutions of the Maxwell's equations in their indefinite forms"

\section{MAXWELL'S EQUATIONS IN SYMMETRIC FORM}

Let us introduce hypothetical free magnetic sources into Maxwell's equations and make a notational change in free electrical sources as $\vec{J}_{C}^{e}(\vec{r} ; t)$ : Electrical conduction current density $\left[\mathrm{A} / \mathrm{m}^{2}\right]$ $\rho_{f}^{e}(\vec{r} ; t):$ Free electrical charge density $\left[\mathrm{C} / \mathrm{m}^{3}\right]$ $\vec{J}_{C}^{m}(\vec{r} ; t)$ : Magnetic conduction current density [V/ $\left.\mathrm{m}^{2}\right]$ $\rho_{f}^{m}(\vec{r} ; t)$ : Free magnetic charge density $\left[\mathrm{Wb} / \mathrm{m}^{3}\right]$

Discussions on the availability of isolated magnetic poles are beyond the interest of the present work (cf.[19]). However, the concept of magnetic conductors is commonly used in electromagnetic radiation problems. Accordingly, the corresponding pairs are outlined as follows:

\section{Form B1.}

Fundamental Fields: $(\vec{E}, \vec{B})$

Fundamental Sources: $\left(\rho_{f}^{e}, \vec{J}_{C}^{e}\right),\left(\rho_{P}^{e}, \vec{J}_{P}^{e}\right), \vec{J}_{V O}^{m},\left(\rho_{f}^{m}, \vec{J}_{C}^{m}\right)$ 
$\operatorname{curl} \vec{E}+\frac{\partial \vec{B}}{\partial t}=-\vec{J}_{C}^{m}, \quad \operatorname{curl} \vec{B}-\frac{1}{c_{0}^{2}} \frac{\partial \vec{E}}{\partial t}=\mu_{0}\left(\vec{J}_{C}^{e}+\vec{J}_{P}^{e}+\vec{J}_{V O}^{m}\right)$

$\operatorname{div} \vec{E}=\left(1 / \varepsilon_{0}\right)\left(\rho_{f}+\rho_{P}^{e}\right) \quad, \operatorname{div} \vec{B}=0$

\section{Form B2.}

Fundamental Fields: $(\vec{E}, \vec{H})$

Fundamental Sources: $\left(\rho_{f}^{e}, \vec{J}_{C}^{e}\right),\left(\rho_{P}^{e}, \vec{J}_{P}^{e}\right),\left(\rho_{P}^{m}, \vec{J}_{P}^{m}\right),\left(\rho_{f}^{m}, \vec{J}_{C}^{m}\right)$

$\operatorname{curl} \vec{E}+\mu_{0} \frac{\partial \vec{H}}{\partial t}=-\vec{J}_{C}^{m}-\vec{J}_{P}^{m}, \operatorname{curl} \vec{H}-\varepsilon_{0} \frac{\partial \vec{E}}{\partial t}=\vec{J}_{C}^{e}+\vec{J}_{P}^{e}$

$\operatorname{div} \vec{E}=\left(1 / \varepsilon_{0}\right)\left(\rho_{f}^{e}+\rho_{P}^{e}\right) \quad, \operatorname{div} \vec{H}=\left(1 / \mu_{0}\right) \rho_{P}^{m}$

\section{Form B3.}

Fundamental Fields: $(\vec{D}, \vec{B})$

Fundamental Sources: $\left(\rho_{f}^{e}, \vec{J}_{C}^{e}\right), \vec{J}_{V O}^{e}, \vec{J}_{V O}^{m},\left(\rho_{f}^{m}, \vec{J}_{C}^{m}\right)$

$\operatorname{curl} \vec{D}+\varepsilon_{0} \frac{\partial \vec{B}}{\partial t}=\varepsilon_{0} \vec{J}_{V O}^{e}, \operatorname{curl} \vec{B}-\mu_{0} \frac{\partial \vec{D}}{\partial t}=\mu_{0}\left(\vec{J}_{C}+\vec{J}_{V O}^{m}\right)$

$\operatorname{div} \vec{D}=\rho_{f}^{e}, \operatorname{div} \vec{B}=0$

Form B4.

Fundamental Fields: $(\vec{D}, \vec{H})$

Fundamental Sources: $\left(\rho_{f}^{e}, \vec{J}_{C}^{e}\right),\left(\rho_{P}^{m}, \vec{J}_{P}^{m}\right), \vec{J}_{V O}^{e},\left(\rho_{f}^{m}, \vec{J}_{C}^{m}\right)$

$\operatorname{curl} \vec{D}+\frac{1}{c_{0}^{2}} \frac{\partial \vec{H}}{\partial t}=\varepsilon_{0}\left(\vec{J}_{V O}^{e}-\vec{J}_{P}^{m}-\vec{J}_{C}^{m}\right), \operatorname{curl} \vec{H}-\frac{\partial \vec{D}}{\partial t}=\vec{J}_{C}^{e}$

$\operatorname{div} \vec{D}=\rho_{f}^{e}, \operatorname{div} \vec{H}=\left(1 / \mu_{0}\right) \rho_{P}^{m}$ 
Remark 9: There is an astonishing coupling mechanism in Maxwell's equations such that electric and magnetic type quantities seem to interchange under a certain rule in passing from one relation to another. It is called the duality principle and the transformations are as follows:

$\vec{E} \rightarrow \vec{H}, \rho_{f}^{e} \rightarrow \rho_{f}^{m}, \vec{J}_{C}^{e} \rightarrow \vec{J}_{C}^{m}, \rho_{P}^{e} \rightarrow \rho_{P}^{m}, \vec{P}^{e} \rightarrow \vec{P}^{m}, \vec{J}_{P}^{e} \rightarrow \vec{J}_{P}^{m}, \vec{J}_{V O}^{e} \rightarrow \vec{J}_{V O}^{m}, \vec{D} \rightarrow \vec{B} ;$

$\vec{H} \rightarrow-\vec{E}, \rho_{f}^{m} \rightarrow-\rho_{f}^{e}, \vec{J}_{C}^{m} \rightarrow-\vec{J}_{C}^{e}, \rho_{P}^{m} \rightarrow-\rho_{P}^{e}, \vec{P}^{m} \rightarrow-\vec{P}^{e}, \vec{J}_{P}^{m} \rightarrow-\vec{J}_{P}^{e}, \vec{J}_{V O}^{m} \rightarrow-\vec{J}_{V O}^{e}, \vec{B} \rightarrow-\vec{D} ;$

$\varepsilon_{0} \leftrightarrow \mu_{0}$ in any field relation to obtain another (the dual) one. This property also implies that the dual transformations in the solution of a certain problem directly yield the solution of the dual problem without effort. When $\rho_{f}^{m}, \vec{J}_{C}^{m}$ are not involved in a model, then the duality principle applies only when $\rho_{f}^{e}, \vec{J}_{C}^{e}$ are zero as well, i.e., in nonconducting media.

Remark 10: Are Maxwell's equations more informative in differential or integral form? This question has been discussed in detail in [20] and concluded that two different formulations of the same phenomenon need to be equally informative (though not supposed to be equally practical in every case) as long as both sets are postulated properly. By equal information we imply that any mathematical relation derived through one set of Maxwell's equations should also be derivable through the other set.

\section{THE PRINCIPLE OF CONTINUITY}

If one applies the divergence operator on (1b) and substitute (1c) into the resultant equation, the field components $\vec{D}(\vec{r} ; t)$ and $\vec{H}(\vec{r} ; t)$ are eliminated to yield

$$
\operatorname{div} \vec{J}_{C}(\vec{r} ; t)+\frac{\partial \rho_{f}}{\partial t}(\vec{r} ; t)=0
$$


which is called "the principle of continuity for conduction currents". (12) can be expressed in words as follows: "The divergence of the density of electrical conduction currents at a point is equal to the time rate of decrease of static charges at the same point". For Maxwell's equations in symmetric form the continuity relations for all types of sources can be outlined as follows:

$$
\operatorname{div} \vec{J}_{C}^{e, m}(\vec{r} ; t)+\frac{\partial \rho_{f}^{e, m}}{\partial t}(\vec{r} ; t)=0, \operatorname{div} \vec{J}_{P}^{e, m}(\vec{r} ; t)+\frac{\partial \rho_{P}^{e, m}}{\partial t}(\vec{r} ; t)=0 \quad(13 \mathrm{a}-\mathrm{d})
$$

The integral form of (13a-d) in an arbitrary volume can be written as

$$
\oint_{\partial \vartheta} \vec{J}_{C}^{e, m} \cdot d \vec{S}+\frac{d}{d t} \int_{\vartheta} \rho_{f}^{e, m} d \vartheta=0, \oint_{\partial \vartheta} \vec{J}_{P}^{e, m} \cdot d \vec{S}+\frac{d}{d t} \int_{\vartheta} \rho_{P}^{e, m} d \vartheta=0
$$

When the Maxwell equations are considered as the fundamental laws of stationary continuous media, then the continuity relations (13) or (14) follow as corollaries. It is also possible to go backwards by postulating the four continuity relations for free and polarized electrical/magnetic currents to derive the four Maxwell equations in symmetric form (see [21]).

\section{A PHYSICAL INTERPRETATION OF THE MAXWELL'S EQUATIONS IN POINT FORM}

A physical interpretation of the relations (1a-d) and (2) can be given when one incorporates the integral form definitions of curl and divergence operators. For a comprehensive discussion on the definitions of vector operators the reader may refer to [22]. Now, assume that we are involved in electromagnetic phenomena taking place at an arbitrary fixed point at the instant. Then one can write

$$
\lim _{\Delta S \rightarrow P} \max \left[\frac{1}{\Delta S} \hat{n} \oint_{\Delta C} \vec{E}(\vec{r} ; t) \cdot d \vec{c}\right]=-\frac{\partial \vec{B}}{\partial t}(P ; t)
$$




$$
\begin{aligned}
& \lim _{\Delta S \rightarrow P} \max \left[\frac{1}{\Delta S} \hat{n} \oint_{\Delta C} \vec{H}(\vec{r} ; t) \cdot d \vec{c}\right]=\frac{\partial \vec{D}}{\partial t}(P ; t)+\vec{J}_{C}(P ; t) \\
& \lim _{\Delta \vartheta \rightarrow P} \frac{1}{\Delta \vartheta} \oint_{\Delta \bar{S}} \vec{D}(\vec{r} ; t) \cdot d \vec{S}=\rho_{f}(P ; t) \\
& \lim _{\Delta \vartheta \rightarrow P} \frac{1}{\Delta \vartheta} \oint_{\Delta \bar{S}} \vec{B}(\vec{r} ; t) \cdot d \vec{S}=0 \\
& \lim _{\Delta \vartheta \rightarrow P} \frac{1}{\Delta \vartheta} \oint_{\Delta \bar{S}} \vec{J}_{C}(\vec{r} ; t) \cdot d \vec{S}=-\frac{\partial \rho_{f}}{\partial t}(P ; t) .
\end{aligned}
$$

In $(15 \mathrm{a}, \mathrm{b})$ we introduce an arbitrary infinitisimal open surface $\Delta S$ with unit normal $\hat{n}$ and enclosure $\Delta C$ that includes and shrinks onto a fixed point $P$ in the limiting case. The direction of $\hat{n}$ and $d \vec{c}$ are specified according to the right hand rule. In $(15 \mathrm{c}, \mathrm{d})$ to (16) we have an arbitrary infinitisimal volume $\Delta \vartheta$ with enclosure $\Delta \bar{S}$ that also includes and shrinks onto the fixed point $P$ in the limiting case (see Fig.s 1 and 2).

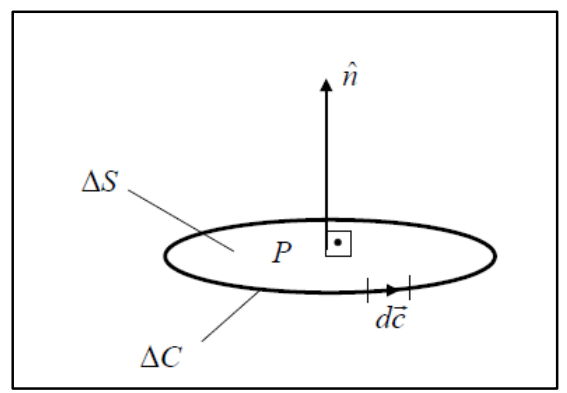

Figure 1

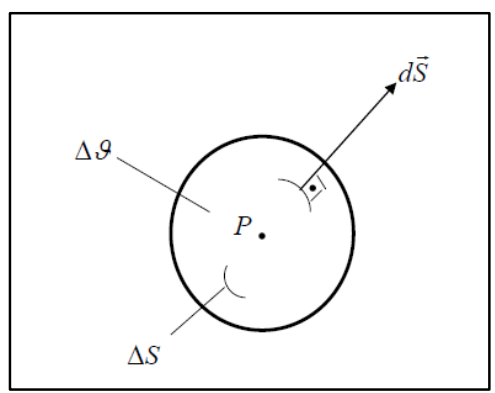

Figure 2

\subsection{An Interpretation of Faraday's Law in Point Form}

We perform contour integrations of the total electrical field around the enclosures of infinitely many infinitisimal open fixed surfaces (as in Fig. 1) defined by the only condition that they include (and shrink in the limit onto) the fixed point $\mathrm{P}$ and divide the result to the surface area. Each time we obtain a real number. However, there is 
only one open surface of them all that yields the greatest value. That numerical value together with the accompanying unit normal vector of that surface describe the curl of the electrical field at the point $\mathrm{P}$, which is also equal to the time rate of decrease of the total magnetic displacement field at the same point. We understand that the direction and magnitude of the vector $\frac{\partial \vec{B}}{\partial t}$ at any point yields information about the "maximum circulation" of the electrical field at the same point. To be more specific we shall denote the surface, its enclosure and the unit normal that maximize the contour integration by $\Delta S_{M}, \Delta C_{M}$ and $\hat{n}_{M}$, respectively. Then our relation can be written as

$$
\lim _{\Delta S_{M} \rightarrow P} \frac{1}{\Delta S_{M}} \hat{n}_{M} \oint_{\Delta C_{M}} \vec{E}(\vec{r} ; t) \bullet d \vec{c}=-\frac{\partial \vec{B}}{\partial t}(P ; t)
$$

which yields

$$
\frac{\partial \vec{B}}{\partial t}(P ; t) \| \hat{n}_{M} \quad \text { and } \quad\left|\frac{\partial \vec{B}}{\partial t}(P ; t)\right|=\lim _{\Delta S_{M} \rightarrow P} \frac{1}{\Delta S_{M}}\left|\oint_{\Delta C_{M}} \vec{E}(\vec{r} ; t) \cdot d \vec{c}\right| \text {. }
$$

A further manipulation on the integral representation is also possible: One may apply scalar product operation $\Delta S_{M} \hat{n}_{M} \cdot$ to each side to get

$$
\lim _{\Delta C_{M} \rightarrow P} \oint_{\Delta C_{M}} \vec{E}(\vec{r} ; t) \cdot d \vec{c}=-\lim _{\Delta S_{M} \rightarrow P} \frac{\partial \vec{B}}{\partial t}(P ; t) \cdot \hat{n}_{M} \Delta S_{M}
$$

and rearrange the right hand side as

$$
\begin{aligned}
\lim _{\Delta S_{M} \rightarrow P} \frac{\partial \vec{B}}{\partial t}(P ; t) \cdot \hat{n}_{M} \Delta S_{M} & =\lim _{\Delta S_{M} \rightarrow P} \int_{\Delta S_{M}} \frac{\partial \vec{B}}{\partial t}(\vec{r} ; t) \cdot d \vec{S}=\lim _{\Delta S_{M} \rightarrow P} \frac{d}{d t} \int_{\Delta S_{M}} \vec{B}(\vec{r} ; t) \cdot d \vec{S} \\
& =\frac{d}{d t} \lim _{\Delta S_{M} \rightarrow P} \psi_{m}\left(\Delta S_{M} ; t\right)=\frac{d \psi_{m}}{d t}(P ; t)
\end{aligned}
$$


where $\quad$ we introduce $\quad \psi_{m}\left(\Delta S_{M} ; t\right)=\int_{\Delta S_{M}} \vec{B}(\vec{r} ; t) \cdot d \vec{S} \quad$ and $\lim _{\Delta S_{M} \rightarrow P} \psi_{m}\left(\Delta S_{M} ; t\right)=\psi_{m}(P ; t)$ as "the instantaneous total magnetic flux passing through the surface $\Delta S_{M}$ and point $P$, respectively. It should be noted that $\psi_{m}\left(\Delta S_{M} ; t\right)$ and $\psi_{m}(P ; t)$ and quantities with similar arguments that follow are nonstandard notations introduced by the author. In the theory of analytical functions the formal equivalence $\lim _{\Delta S_{M} \rightarrow P} \frac{\partial \vec{B}}{\partial t}(P ; t) \cdot \hat{n}_{M} \Delta S_{M}=\lim _{\Delta S_{M} \rightarrow P} \int_{\Delta S_{M}} \frac{\partial \vec{B}}{\partial t}(\vec{r} ; t) \cdot d \vec{S} \quad$ is known to hold when $\frac{\partial \vec{B}}{\partial t}(\vec{r} ; t)$ remains finite (bounded) on the surface $\Delta S_{M}$.

The term $\oint_{\Delta C_{M}} \vec{E}(\vec{r} ; t) \cdot d \vec{c}$ addresses the total work done by the (electrical) sources in carrying a (free) $+1 C$ electrical point charge around the fixed contour $\Delta C_{M}$ as observed by the electrical point charge itself. It is also called "the electromotive force" ("emf") acting on $\Delta S_{M}$, again, as observed by the point charge. We shall call its limiting value as $\Delta C_{M} \rightarrow P$ as "the instantaneous electromotive force at point $P$ ", or $\operatorname{emf}(P ; t)$.

Consequently, Faraday's Law in point form can be stated alternative to (15a) as

$\operatorname{emf}(P ; t)=-\frac{d \psi_{m}}{d t}(P ; t)$

and expressed in words as "the instantaneous electromotive force at a fixed point $P$ is equal to the time rate of decrease total magnetic flux passing through that point". Obviously, the directions of circulation and the normal of the surface are always specified in accord with the right hand rule unless otherwise stated. 
When electrical and magnetic fields remain finite inside any neighborhood of the point $P$, the individual terms in (17) vanish in a uniform manner in the limiting case $\Delta S_{M} \rightarrow P$ to yield

$$
\operatorname{emf}(P ; t)=0, \quad \psi_{m}(P ; t)=0 .
$$

Obviously, the new point form quantities $\operatorname{emf}(P ; t)$ and $\psi_{m}(P ; t)$ make sense (are nontrivial) when singular electrical and/or magnetic field quantities are involved.

\subsection{An Interpretation of Ampere's Law in Point Form}

Based on our expertise with Faraday's Law we can express Ampere's Law directly as follows: "The maximum circulation of the magnetic field around a fixed point $P$ is equal to the total current density (sum of displacement and conduction current densities) passing through that point" or

$$
\lim _{\Delta S_{M} \rightarrow P} \frac{1}{\Delta S_{M}} \hat{n}_{M} \oint_{\Delta C_{M}} \vec{H}(\vec{r} ; t) \cdot d \vec{c}=\frac{\partial \vec{D}}{\partial t}(P ; t)+\vec{J}_{C}(P ; t)
$$

which yields

$$
\frac{\partial \vec{D}}{\partial t}(P ; t)+\vec{J}_{C}(P ; t) \| \hat{n}_{M}
$$

and

$$
\left|\frac{\partial \vec{D}}{\partial t}(P ; t)+\vec{J}_{C}(P ; t)\right|=\lim _{\Delta S_{M} \rightarrow P} \frac{1}{\Delta S_{M}}\left|\oint_{\Delta C_{M}} \vec{H}(\vec{r} ; t) \cdot d \vec{c}\right|
$$

One should note that $\Delta S_{M}, \Delta C_{M}$ and $\hat{n}_{M}$ in Ampere's Law is by no means related to those calculated in Faraday's Law and is only a matter of notation.

A further manipulation as before gives 
$\lim _{\Delta C_{M} \rightarrow P} \oint_{\Delta C_{M}} \vec{H}(\vec{r} ; t) \cdot d \vec{c}=\lim _{\Delta S_{M} \rightarrow P}\left[\frac{\partial \vec{D}}{\partial t}(P ; t)+\vec{J}_{C}(P ; t)\right] \cdot \hat{n}_{M} \Delta S_{M}$

The right hand side can be arranged into

$$
\begin{aligned}
\lim _{\Delta S_{M} \rightarrow P}\left[\frac{\partial \vec{D}}{\partial t}(P ; t)+\vec{J}_{C}(P ; t)\right] \cdot \hat{n}_{M} \Delta S_{M} & =\lim _{\Delta S_{M} \rightarrow P}\left[\int_{\Delta S_{M}} \frac{\partial \vec{D}}{\partial t}(\vec{r} ; t) \cdot d \vec{S}+\int_{\Delta S_{M}} \vec{J}_{C}(\vec{r} ; t) \cdot d \vec{S}\right] \\
& =\lim _{\Delta S_{M} \rightarrow P}\left[\frac{d \psi_{e}}{d t}\left(\Delta S_{M} ; t\right)+I_{C}\left(\Delta S_{M} ; t\right)\right] \\
& =\frac{d \psi_{e}}{d t}(P ; t)+I_{C}(P ; t)
\end{aligned}
$$

where we introduce $\psi_{e}\left(\Delta S_{M} ; t\right)=\int_{\Delta S_{M}} \vec{D}(\vec{r} ; t) \cdot d \vec{S} \quad$ as "the instantaneous total electrical flux passing through the surface $\Delta S_{M}$ " and $I_{C}(P ; t)=\lim _{\Delta S_{M} \rightarrow P} I_{C}\left(\Delta S_{M} ; t\right)=\lim _{\Delta S_{M} \rightarrow P} \int_{\Delta S_{M}} \vec{J}_{C}(\vec{r} ; t) \cdot d \vec{S} \quad$ as "the instantaneous free current passing through point $P$ ". Due to duality between Faraday's and Ampere's Laws, the term $\oint_{\Delta C_{M}} \vec{H}(\vec{r} ; t) \cdot d \vec{c}$ addresses the total work done by the sources in carrying a (free) $+1 \mathrm{~Wb}$ magnetic point charge around the fixed contour $\Delta C_{M}$ as observed by the magnetic point charge itself. This terms is also called "the magnetomotive force" ("mmf") acting on $\Delta S_{M}$, again, as observed by the magnetic point charge itself. We shall call its limiting value as $\Delta C_{M} \rightarrow P$ as "the instantaneous magnetomotive force at point $P$ ", or $\operatorname{mmf}(P ; t)$. Please note that $\mathrm{mmf}$ is a physical (measurable) quantity although it has been described via the fictitious magnetic point charge not included in our fundamental representation of the field equations (1a-d).

In the limiting case $\Delta S_{M} \rightarrow P$ the alternative representation of Ampere's Law can be given as 
$\operatorname{mmf}(P ; t)=\psi_{e}(P ; t)+I_{C}(P ; t)$

which can be expressed in words as "the instantaneous magnetomotive force at a point $P$ is equal to the sum of the total instantaneous electric flux and the free currents passing through that point". Again, the point form quantities in (18) are nontrivial only when $\vec{H}(\vec{r} ; t), \vec{D}(\vec{r} ; t)$ and $\vec{J}_{C}(\vec{r} ; t)$ display singular behavior at point $P$. As one of the simplest cases $\vec{J}_{C}(\vec{r} ; t)$ can be observed to have a singular nature at a point $P$ when a uniform current flows along a curve, or on a surface that includes that point.

\subsection{An Interpretation of the Law of Conservation of Electrical Flux in Point Form}

In (15c) we first multiply each side by $\Delta \vartheta$ to get

$$
\lim _{\Delta \bar{S} \rightarrow P} \oint_{\Delta \vec{S}} \vec{D}(\vec{r} ; t) \cdot d \vec{S}=\lim _{\Delta \vartheta \rightarrow P} \rho_{f}(P ; t) \Delta \vartheta
$$

Each side can be put into the final forms

$$
\begin{aligned}
& \lim _{\Delta \bar{S} \rightarrow P} \oint_{\Delta \bar{S}} \vec{D}(\vec{r} ; t) \cdot d \vec{S}=\lim _{\Delta \bar{S} \rightarrow P} \psi_{e}(\Delta \bar{S} ; t)=\psi_{e}(P ; t), \\
& \lim _{\Delta \vartheta \rightarrow P} \int_{\Delta \vartheta} \rho_{f}(\vec{r} ; t) d \vartheta=\lim _{\Delta \vartheta \rightarrow P} Q(\Delta \vartheta ; t)=Q(P ; t)
\end{aligned}
$$

to get

$\psi_{e}(P ; t)=Q(P ; t)$,

or "the total instantaneous electrical flux emerging from any point is equal to the total instantaneous electrical charge located on the same point". It should be emphasized that $Q(P ; t)$ in (19) is nontrivial only when $\rho_{f}(\vec{r} ; t)$ displays singular behavior at point $P$. For instance, this case may easily be associated with an isolated point charge located at that point. However, if we consider a uniform distribution of line and surface charges on a curve $C$ and a surface $\Sigma$ that cross 
the point $P$ with arbitrary but finite valued (line and surface) charge densities $\rho_{L}(\vec{r} ; t)$ and $\rho_{S}(\vec{r} ; t)$, then volume integrations in an infinitisimal region $\Delta \vartheta$ that contains and shrinks onto point $P$ reduce as

$$
\begin{aligned}
\lim _{\Delta \vartheta \rightarrow P} \int_{\Delta \vartheta} \rho_{f}(\vec{r} ; t) d \vartheta & =\lim _{\Delta C \rightarrow P} \int_{\Delta C} \rho_{L}(\vec{r} ; t) d L \\
\cdot \lim _{\Delta \vartheta \rightarrow P} \int_{\Delta \vartheta} \rho_{f}(\vec{r} ; t) d \vartheta & =\lim _{\Delta \Sigma \rightarrow P} \int_{\Delta \Sigma} \rho_{S}(\vec{r} ; t) d S .
\end{aligned}
$$

Due to a standard theorem of integration, the right hand side integrals have values in the range

$$
\begin{aligned}
& \lim _{\Delta C \rightarrow P} \min _{\vec{r} \in \Delta C} \rho_{L}(\vec{r} ; t) \Delta C \leq \lim _{\Delta C \rightarrow P} \int_{\Delta C} \rho_{L}(\vec{r} ; t) d L \leq \lim _{\Delta C \rightarrow P} \max _{\vec{r} \in \Delta C} \rho_{L}(\vec{r} ; t) \Delta C \\
& \lim _{\Delta \Sigma \rightarrow P} \min _{\vec{r} \in \Delta C} \rho_{S}(\vec{r} ; t) \Delta \Sigma \leq \lim _{\Delta \Sigma \rightarrow P} \int_{\Delta \Sigma} \rho_{S}(\vec{r} ; t) d S \leq \lim _{\Delta \Sigma \rightarrow P} \max _{\vec{r} \in \Delta \Sigma} \rho_{S}(\vec{r} ; t) \Delta \Sigma,
\end{aligned}
$$

the end points of which go to zero uniformly. Here we assume $\rho_{L}(\vec{r} ; t)$ or $\rho_{S}(\vec{r} ; t)$ to have a positive sign. A similar procedure applies when any of them has negative sign. This example demonstrates that $Q(P ; t)=0$ for these two geometries.

\subsection{An Interpretation of the Law of Conservation of Magnetic Flux in Point Form}

Due to the similar structure of (15d) with (15c), this law can be expressed in a straightforward manner as

$\psi_{m}(P ; t)=0$,

or "the total instantaneous magnetic flux emerging from any point is equal zero", which, again, is nontrivial only when $\vec{B}(\vec{r} ; t)$ displays singular behavior at point $P$. 


\subsection{An Interpretation of the Law of Continuity of Free Currents in Point Form}

We first multiply (16) by $\Delta \vartheta$ to get

$$
\lim _{\Delta \bar{S} \rightarrow P} \oint_{\Delta \bar{S}} \vec{J}_{C}(\vec{r} ; t) \cdot d \vec{S}=-\lim _{\Delta \vartheta \rightarrow P} \frac{\partial \rho_{f}}{\partial t}(P ; t) \Delta \vartheta
$$

and then put each side of this relation into the final forms

$$
\begin{aligned}
\lim _{\Delta \bar{S} \rightarrow P} \oint_{\Delta \bar{S}} \vec{J}_{C}(\vec{r} ; t) \cdot d \vec{S} & =\lim _{\Delta \bar{S} \rightarrow P} I_{C}(\Delta \bar{S} ; t)=I_{C}(P ; t), \\
\lim _{\Delta V \rightarrow P} \frac{\partial \rho_{f}}{\partial t}(P ; t) \Delta \vartheta & =\lim _{\Delta \vartheta \rightarrow P} \int_{\Delta \vartheta} \frac{\partial \rho_{f}}{\partial t}(\vec{r} ; t) d \vartheta \\
& =\frac{d}{d t} \lim _{\Delta \vartheta \rightarrow P} \int_{\Delta \vartheta} \rho_{f}(\vec{r} ; t) d \vartheta=\frac{d Q}{d t}(P ; t)
\end{aligned}
$$

to get

$$
I_{C}(P ; t)=-\frac{d Q}{d t}(P ; t),
$$

which can be interpreted as "the total instantaneous free current emerging from any point is equal to the time rate of decrease of the total instantaneous charge at the same point".

A similar interpretation also applies to electrical and magnetic polarization currents:

$$
I_{P}^{e, m}(P ; t)=-\frac{d Q_{P}^{e, m}}{d t}(P ; t),
$$

where we may describe the instantaneous electrical/magnetic polarization charges and currents at an arbitrary point in terms of the instantaneous electrical/magnetic polarization flux $\psi_{P}^{e, m}(P ; t)$ at the same point through 


$$
\begin{aligned}
I_{P}^{e, m}(P ; t)=\lim _{\Delta \bar{S} \rightarrow P} \oint_{\Delta \bar{S}} \vec{J}_{P}^{e, m}(\vec{r} ; t) \cdot d \vec{S} & =\lim _{\Delta \bar{S} \rightarrow P} \oint_{\Delta \bar{S}} \frac{\partial \vec{P}^{e, m}}{\partial t}(\vec{r} ; t) \cdot d \vec{S} \\
& =\frac{d}{d t} \lim _{\Delta \bar{S} \rightarrow P} \oint_{\Delta \bar{S}} \vec{P}^{e, m}(\vec{r} ; t) \cdot d \vec{S}=\frac{d \psi_{P}^{e, m}}{d t}(P ; t) \\
Q_{P}^{e, m}(P ; t)=\lim _{\Delta \vartheta \rightarrow P} \int_{\Delta \vartheta} \rho_{P}^{e, m}(\vec{r} ; t) d \vartheta & =-\lim _{\Delta \vartheta \rightarrow P} \int_{\Delta \vartheta} \operatorname{div} \vec{P}^{e, m}(\vec{r} ; t) d \vartheta \\
& =-\lim _{\Delta \bar{S} \rightarrow P} \oint_{\Delta \bar{S}} \vec{P}^{e, m}(\vec{r} ; t) \bullet d \vec{S}=-\psi_{P}^{e, m}(P ; t)
\end{aligned}
$$

\subsection{A Special Case: "The current at the tip of an infinitely thin conducting wire"}

We shall consider an "infinitely thin" arbitrary conducting wire carrying a dynamic free current $I_{C}(\vec{r}, t)$ (see Fig. 3). Let us assume that this thin wire is cut abruptly at two tip points, one of which we shall call $P$. By a "flux integration" of the Faraday's Law (1b) through an infinitely small closed surface $\Delta \bar{S}$ that shrinks onto the fixed point $P$, we shall show that $I_{C}(P ; t)=0$.

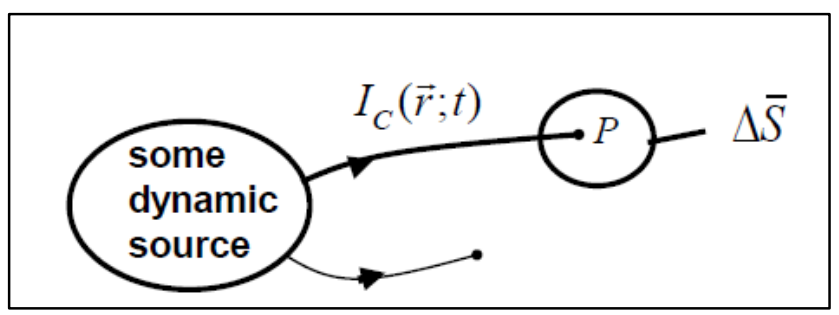

Figure 3

A "flux integration" of the Faraday's Law through any arbitrary closed surface $\bar{S}$ is written by

$$
\oint_{\bar{S}} \operatorname{curl} \vec{H}(\vec{r} ; t) \cdot d \vec{S}=\oint_{\bar{S}} \frac{\partial \vec{D}}{\partial t}(\vec{r} ; t) \cdot d \vec{S}+\oint_{\vec{S}} \vec{J}_{C}(\vec{r} ; t) \cdot d \vec{S}
$$

Due to Stokes' theorem, the left hand side equals to the circulation of the magnetic field around the enclosure of $\bar{S}$. However, $\bar{S}$ is $a$ priori a closed surface and, by definition, has a zero enclosure. 
Therefore, assuming that $\vec{H}(\vec{r} ; t)$ does not possess any singularity at any point on $\bar{S}$, the left hand side always yields zero:

$$
\oint_{\vec{S}} \operatorname{curl} \vec{H}(\vec{r} ; t) \cdot d \vec{S}=0 \quad, \quad \oint_{\vec{S}} \frac{\partial \vec{D}}{\partial t}(\vec{r} ; t) \cdot d \vec{S}=-\oint_{\vec{S}} \vec{J}_{C}(\vec{r} ; t) \cdot d \vec{S} .
$$

Next we consider the closed surface to be infinitely small and shrink onto the fixed point $P$ in the limit. Then one writes

$$
\lim _{\Delta \vec{S} \rightarrow P} \oint_{\bar{S}} \frac{\partial \vec{D}}{\partial t}(\vec{r} ; t) \cdot d \vec{S}=-\lim _{\Delta \vec{S} \rightarrow P} \oint_{\bar{S}} \vec{J}_{C}(\vec{r} ; t) \cdot d \vec{S} .
$$

The right hand side formally yields $I_{C}(P ; t)$ and the right hand side yields zero through (19) as

$\lim _{\Delta \bar{S} \rightarrow P} \oint_{\Delta \bar{S}} \frac{\partial \vec{D}}{\partial t}(\vec{r} ; t) \cdot d \vec{S}=\frac{d}{d t}\left[\lim _{\Delta \bar{S} \rightarrow P} \oint_{\Delta \vec{S}} \vec{D}(\vec{r} ; t) \cdot d \vec{S}\right]=\frac{d \psi_{e}}{d t}(P ; t)=\frac{d Q}{d t}(P ; t)=0$, since $Q(P ; t)=0$ as described following (19). This result is based on the physical fact that only a uniform distribution of line charges can be supported on a simple conductor. Alternatively, this can also be taken as a mathematical description for a simple conductor. So we obtain the desired result which is usually incorporated as a boundary condition in modeling of thin wire antennas.

\section{CONCLUSION}

In the present work a flowchart for analytical investigtion of electromagnetic phenomena is sketched and the axiomatic structure of classical electromagnetic theory based on Maxwell's equations is studied in stationary continuous media from a Hertzian perspective. The extension of the present subject for moving bodies is available in the authors's earlier work [11] on the axiomatic structure of Hertzian electrodynamics. 


\section{REFERENCES}

[1] G.T. Gillies, Ed. Special Issue on Electrical Charge, Metrologia, 41 (2004).

[2] P. Heering. "On Coulomb's Inverse Square Law", American Journal of Physics Vol.60, No.1, pp.988-994, November (1992).

[3] D.J. Griffiths. Introduction to Electrodynamics, Prentice Hall, Inc. (1989).

[4] R.M. Fano, L.J.Chu and R.B. Adler. Electromagnetic Fields, Energy, and Forces, John Wiley \& Sons, Inc. (1960).

[5] L.M. Magid. Electromagnetic Fields, Energy and Waves, John Wiley and Sons, Inc., (1972).

[6] P.C. Clemmov. An Introduction to Electromagnetic Theory, Cambridge University Press, (1973).

[7] J.D. Jackson. Classical Electrodynamics, 3rd Ed., John Wiley and Sons Inc., (1999)

[8] J.C. Maxwell. "A Dynamical Theory of the Electromagnetic Field", Royal Society Transactions 155, pp.459-512 (1865).

[9] T.W. Barrett. "Electromagnetic Phenomena not Explained by Maxwell's Equations", pp.6-86, Essays on the Formal Aspects of Electromagnetic Theory, Ed. A. Lakhtakia, World Scientific Publ. Co., (1993).

[10]O. Heaviside. Electromagnetic Theory Vol.1. "The Electrician" Printing and Publishing Company, Ltd. London (1893).

[11]B. Polat. "On the Axiomatic Structure of Hertzian Electrodynamics" TWMS Journal of Applied and Engineering Mathematics Vol.2 No.1, pp.35-59 (2012).

[12] A.E. Chubykalo and R. Smirnov-Rueda. "Convection Displacement Current and Generalized Form of Maxwell-Lorentz Equations". Modern Physics Letters A, 12 (1). pp. 1-24 (1997). 
[13] A.E. Chubykalo, H.A. Munera and R. Smirnov-Rueda. "Is the Free Electromagnetic Field a Consequence of Maxwell's Equations or a Postulate”. Foundations of Physics Letters, Vol.11 No.6 pp. 573-584 (1998).

[14] J.E. Gray. "Electromagnetic Waves with E parallel to B" J.Phys. A: Math. Gen. 25. pp. 5373-5376, (1992).

[15]Z. Zaghloul and H.A. Buckmaster. "Transverse Electromagnetic Fields with ", pp.183-206, Essays on the Formal Aspects of Electromagnetic Theory, Ed. A. Lakhtakia, World Scientific Publ. Co., (1993).

[16]R. Rojanski, Electromagnetic Fields and Waves, 2nd Ed., Dover Publ. (1979).

[17] A. Shadowitz, The Electromagnetic Field, Dover Publ. (1975).

[18]C.T. Tai, "A Study of Electrodynamics of Moving Media", Proceedings of the IEEE, pp.685-698, 1361-1363, (1964).

[19]M.G. Guillemot. "Completing Maxwell's Equations by Symmetrization". Europhysics Papers, Vol.53 No.2 pp. 155-161 (2001).

[20]B. Polat. "Remarks on the Fundamental Postulates on Field Singularities in Electromagnetic Theory", IEEE Antennas and Propagation Magazine, 47 No.5, 47-54 (2005).

[21]J.A. Heras. "Can Maxwell's Equations Be Obtained from the Continuity Equation?" American Journal of Physics Vol.75, No.7, pp.652-657, July (2007).

[22]B. Polat. "Remarks on Standard Textbook Definitions of Vector Operators", Beykent University, Journal of Science and Engineering (BUJSE), Vol. 7 No.2 83-104 (2014). 